\title{
Automatic Detection Technology of Sonar Image Target Based on the Three-Dimensional Imaging
}

\author{
Wanzeng Kong, ${ }^{1}$ Jinshuai Yu, ${ }^{1}$ Ying Cheng, ${ }^{1}$ Weihua Cong, ${ }^{2}$ and Huanhuan Xue ${ }^{2}$ \\ ${ }^{1}$ College of Computer Science, Hangzhou Dianzi University, Hangzhou, China \\ ${ }^{2}$ Hangzhou Institute of Applied Acoustics, Hangzhou, China \\ Correspondence should be addressed to Wanzeng Kong; kongwanzeng@hdu.edu.cn
}

Received 28 April 2017; Revised 25 July 2017; Accepted 20 August 2017; Published 11 October 2017

Academic Editor: Paolo Mercorelli

Copyright (c) 2017 Wanzeng Kong et al. This is an open access article distributed under the Creative Commons Attribution License, which permits unrestricted use, distribution, and reproduction in any medium, provided the original work is properly cited.

\begin{abstract}
With 3D imaging of the multisonar beam and serious interference of image noise, detecting objects based only on manual operation is inefficient and also not conducive to data storage and maintenance. In this paper, a set of sonar image automatic detection technologies based on 3D imaging is developed to satisfy the actual requirements in sonar image detection. Firstly, preprocessing was conducted to alleviate the noise and then the approximate position of object was obtained by calculating the signal-to-noise ratio of each target. Secondly, the separation of water bodies and strata is realized by maximum variance between clusters (OTSU) since there exist obvious differences between these two areas. Thus image segmentation can be easily implemented on both. Finally, the feature extraction is carried out, and the multidimensional Bayesian classification model is established to do classification. Experimental results show that the sonar-image-detection technology can effectively detect the target and meet the requirements of practical applications.
\end{abstract}

\section{Introduction}

In the Second World War, the US Navy successfully escaped the Japanese seabed minefield by using the sonar equipment. Subsequently, many countries have begun to pay attention to the development of sonar technology, especially in the military field. With the increasing marine development, the exploration of the ocean was limited not only to military purposes but also to commercial and civilian purposes, such as submarine resource development, oil exploration, automatic mapping of submarine topography, and detection of fish stocks. In order to adapt to the underwater environment, intelligent underwater robot research has been carried out for the laying of submarine cables, underwater demining, and so on. As the current requirements for intelligent sonar equipment are getting higher and higher, now there are many underwater target recognition technology applications. Therefore, whether in the field of military or civilian, underwater target recognition technology will be one of the main technologies in the future of ship and ocean engineering to research.
Due to the complexity of the seabed environment, there are some problems such as poor contrast of the sonar image, the serious noise of the submarine reverberation, low resolution, strong interference, and less dark pixels [1], in addition to the object's inherent characteristics of reflecting sound waves which result in the incomplete target edge. Since there is too much noise in the sonar image, it is difficult to ensure the correctness of submarine target recognition if we only consider the visual point. As a result, it is easy to produce false alarms. Therefore, the development of a set of automatic detection technologies for sonar images which has low false alarm rate and high detection rate is particularly important [2].

Casselman et al. proposed a method of multilayer perceptual neural network detection with low false alarm rate and applied this method to the passive sonar detection [3]; Weber and Kruger studied the passive sonar lofar grams contourenhancing technique and contrast enhancement technique based on neural network, which greatly improved the signalto-noise ratio of the image and detected the target signal with signal-to-noise ratio of $-17 \mathrm{~dB}$ with very accurate precision 


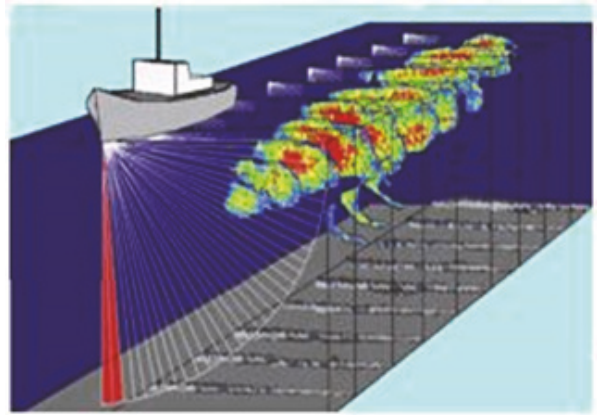

(a)

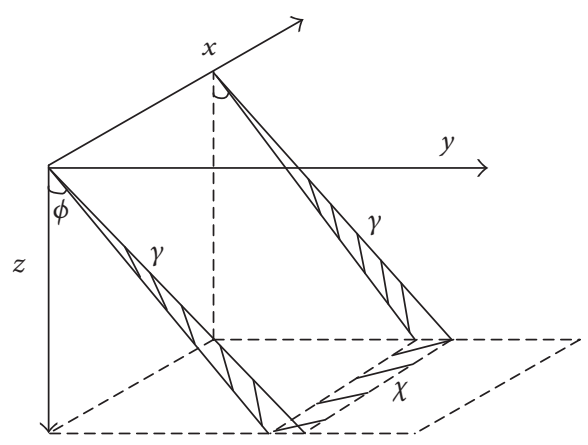

(b)

FIGURE 1: The composition of three-dimensional image data volume.

[4]; Howell and Wood proposed a passive sonar detection and recognition system based on hybrid neural networks [5]; Postolache et al. constructed intelligent passive sonar signal processing systems on Lab VIEW and FPGA platforms [6]. Mill Brown, of the University of Sheffield, UK, studied three time detectors based on the zero-crossing rate of the timedomain signal and the weak underwater acoustic signal was detected. Bertilone and Killeen [7], of the Royal Australian Naval Ship Defense Science and Technology Organization, use the passive band gap generalized energy detection hybrid model to detect the underwater noise, and its false alarm rate is reduced to less than $1 \%$. Compared with the traditional energy detection method, the threshold is reduced by $8 \mathrm{~dB}$.

\section{The Composition of Three-Dimensional Image Data Volume}

The three-dimensional sonar data consists of three dimensions: navigation, distance, and beam. When vessels sail at sea, sonar is used to detect underwater objects. The direction of the sailing vessel is the navigational dimension of the sonar data. The distance from the sonar to the bottom of the sea is the distance dimension of the sonar data. The angle of the acoustic wave divergence in the underwater is the beam dimension of the sonar data. In addition, the distribution of the beam is uniform. Since the threedimensional sonar data can obtain the information of the three-dimensional space object, the image is clearer and more visible than the current image sonar. Three-dimensional sonar data " $\rho(\gamma, \phi, \chi)$ " can transform to $P(x, y, z)$; navigation direction " $\chi$ " is the sequence of equal interval of 8 (sequence length can be adjusted, the maximum is 4096); heading angle $\phi$ is $\left[-40^{\circ}, 40^{\circ}\right]$; echo distance $\gamma$ is the sequence of equal interval (sequence length can be adjusted, the maximum is 4096). Lateral horizontal width $Y=\gamma * \sin \phi$; vertical depth $Z=\gamma * \cos \phi$. Thus, the three-dimensional sonar image is made up of numerous beam's two-dimensional images [8, 9]. The component schematic diagram of three-dimensional sonar data is shown in Figure 1.

\section{Sonar Image Processing}

3.1. Preprocessing and Signal-to-Noise Ratio (SNR). Sonar image preprocessing is the foundation of the sonar image processing and an indispensable part of image recognition [10-13]. Image preprocessing mainly includes image denoising and enhancement. The function of sonar image preprocessing must be maximum to eliminate the influence of all kinds of noise, reduce the influence of noise on the target area, and, at the same time, strengthen the real target image in the water and the part of interest. The background noise of sonar image is divided into three categories, respectively, the environmental noise, reverberation noise, and white noise. In this paper, the sonar image preprocessing includes two steps: first, image denoising; second, bleaching process of sonar signal.

Image denoising methods mainly include neighborhood averaging method, self-adaptive smooth filtering, wavelet transformation denoising method, and median filtering denoising. According to the characteristics of the noise, we can choose one or more targeted denoising methods. In this paper, through the experimental analysis and judgment, we choose the median filtering method to the sonar image processing. The median filter is a kind of nonlinear filters; it can eliminate noise and at the same time keep the detail of the image. The steps are as follows:

(1) Moving the template in turn in the image until the center of the template overlaps with a pixel in the image

(2) Extracting the gray value of the pixels which correspond with the template

(3) Aligning these value in order

(4) Assigning the intermediate value or the average of the two middle values to the central pixel in the corresponding template.

In order to eliminate the isolated noise points in the image, we can make the approximate values replace the pixel values that are significantly different from the surrounding pixels by using the median filter.

Bleaching process of sonar signal refers to the balanced background which can reduce the interference of noise to a certain extent so that the data will be easy to deal with for subsequent processing. According to the characteristics 
and properties of the sonar, choice of sonar signal bleaching processing formula is as follows:

$$
\begin{aligned}
& X(t)=\int_{t-T / 2}^{t+T / 2}|x(t)|^{4 / 5} d t \\
& y(t)=\frac{x(t)}{X(t)} .
\end{aligned}
$$

$x(t)$ refers to data of navigation direction or echo distance. $T$ is the size of the window. Through the bleaching process, the noise signal is weakened obviously which is in favor of the image target recognition.

The signal-to-noise ratio is the ratio of the power spectrum of the signal to the noise, but power spectra are usually hard to measure. Therefore we can use the ratio of signal variance to noise variance to approximate the signal-to-noise ratio. According to the characteristics of sonar image, we can use the following formula to calculate the target's signal-tonoise ratio:

$$
\mathrm{SNR}=10 * \log \frac{\bar{S}-E_{n}}{V_{\text {arn }}} .
$$

$\bar{S}$ is the pixel integral value of the target area in sonar image, $E_{n}$ is the local mean in the target area, and $V_{\text {arn }}$ is the standard deviation of background in the target area. The larger the signal-to-noise ratio in the suspicious area, the greater the chance that the area turns out to be the true target. Sonar target detection technology calculates the signal-tonoise ratio of the suspicious target area, so as to achieve the purpose which is distinguishing the target and interference noise effectively, and largely avoid the noise interference.

3.2. Image Segmentation Algorithm Analysis. In the actual detection of the ocean, sonar needs not only to detect the object in the water but also to detect objects in the stratum. Due to the large differences between the water and stratum, the detection is much easier since there is less noise in the sea water, while the reverberation of stratum is relatively serious, which causes great difficultly in detecting the target. So we can separate the water and stratum and then process the sonar image in different regions, respectively.

In this paper, we use the OTSU algorithm based on the gray-level histogram to proceed image threshold segmentation. A good result of segmentation is obtained by using the corrosion expansion operator to process closure operation.

OTSU threshold segmentation method uses variance to find the best threshold value between the two kinds of pixels and uses variance between clusters to evaluate the segmentation results. The formula is as follows:

$$
w(R)=\frac{\sum_{p_{\in R}} f\left(x_{1}, y_{1}\right)^{2}}{\operatorname{Num}_{t}}+\frac{\sum_{q_{\epsilon R}} f\left(x_{2}, y_{2}\right)^{2}}{\operatorname{Num}_{\bar{t}}}
$$

$\mathrm{Num}_{t}$ and $\mathrm{Num}_{\bar{t}}$ represent the pixel number of the target area and nontarget area, respectively.

Image segmentation is an important part of sonar image processing $[14,15]$. After the separation of water and stratum, the two parts of the image need to be segmented and then the target area is extracted. Since there is less noise in the water, there are many methods available, such as OTSU, iterative threshold method, and two-dimensional maximum entropy threshold segmentation. After experimental analysis, we use the iterative threshold method for threshold selection compared with two-dimensional maximum entropy threshold segmentation. The iterative method is based on the idea of approximation, and its steps are as follows:

(5) The maximum grayscales value and the minimum grayscales value of the image are denoted as $P_{\max }$ and $P_{\min }$, and the initial threshold value $T_{0}=\left(P_{\max }+\right.$ $\left.P_{\min }\right) / 2$.

(6) According to the threshold value $T_{(k)}(k=0,1$, $2, \ldots, k)$, the image is divided into foreground and background, and mean gray values of the two parts are denoted as $\mathrm{H}_{1}$ and $\mathrm{H}_{2}$.

(7) A new threshold value $T_{(k+1)}=\left(H_{1}+H_{2}\right) / 2$ is obtained.

(8) If $T_{(k)}=T_{(k+1)}$, then the result is the threshold; otherwise go to (2), iterative calculation.

Entropy is a function that describes the state of the system and represents the average amount of information. At the same time, entropy is used to calculate the disorder in a system phenomenon and can be used as a measure of the degree of chaos. When performing the evaluation of the segmentation effect, the formula is as follows:

$$
\begin{aligned}
w(R)= & \frac{\sum_{i=0}^{n g} H_{t}(i) \ln H_{t}(i)}{\operatorname{Num}_{t}}-\frac{\sum_{i=0}^{n g} H_{\bar{t}}(i) \ln H_{\bar{t}}(i)}{\operatorname{Num}_{\bar{t}}} \\
& +\ln \operatorname{Num}_{t} \operatorname{Num}_{\bar{t}} .
\end{aligned}
$$

In the sonar image processing, the two-dimensional maximum entropy is often used to divide the threshold to obtain the optimal threshold. The two-dimensional maximum entropy method uses the two-dimensional histogram of pixel intensity and regional gray mean and finds the optimal threshold according to the maximum entropy.

\subsection{Sonar Target Discrimination Based on Multidimensional} Bayesian Classification Model. In fact, we need to not only detect the submarine target but also classify the target preliminary during the sonar sweeping. The main categories are cylindrical, spherical, cable-like targets. In this paper, feature extraction is used to obtain the characteristics of the target. After multiple experiments, the extracted length-width ratio, pixel value, and signal-to-noise ratio are analyzed to find that they conform to the Gaussian distribution. Therefore, this paper adopts a multidimensional Bayesian classification model [8] to classify the target. Bayesian classifier has the advantages of high classification accuracy and generalization ability [16]. At the same time, it does not need a lot of data as the training set. The formula is as follows:

$$
P\left(w_{i} \mid x\right)=\frac{P\left(w_{i} \mid x\right)}{P(x)}
$$




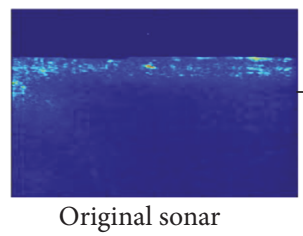

signal

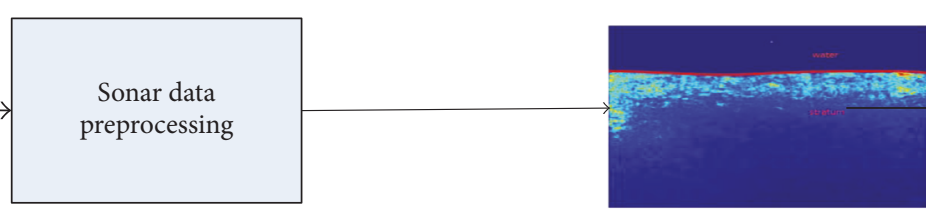

Separate the water and stratum

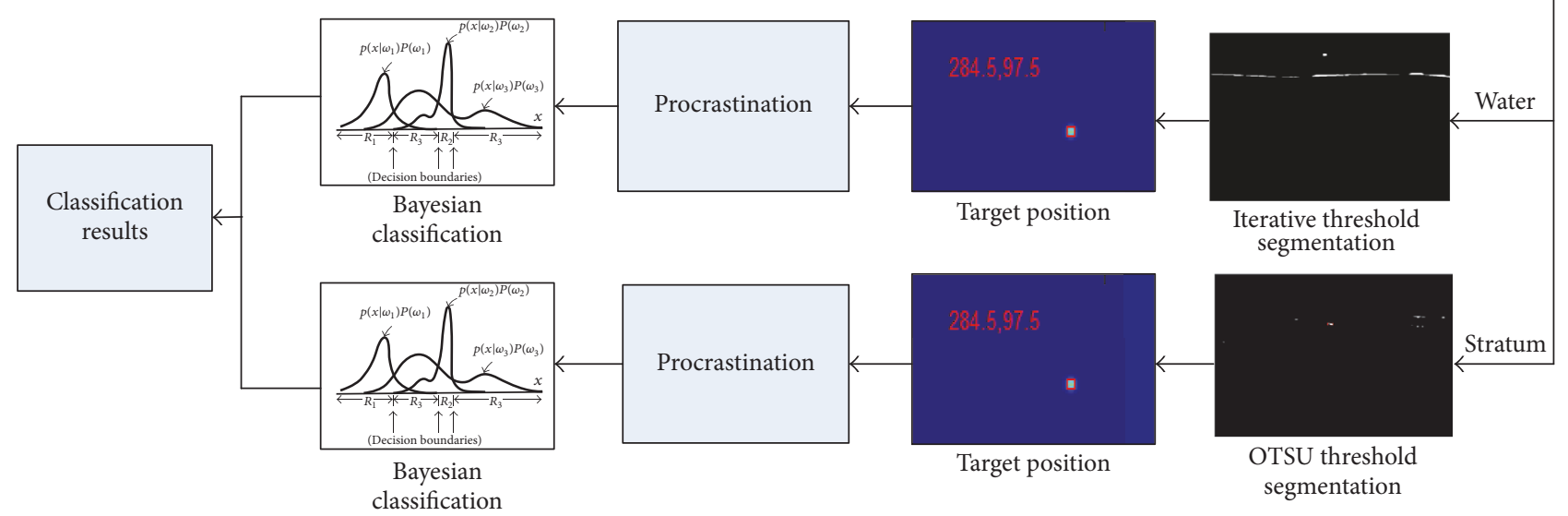

FIGURE 2: Sonar image processing flow chart.

$P\left(x \mid w_{i}\right)$ is the likelihood function, $P\left(w_{i}\right)$ is the prior probability, $\sum_{j} P\left(w_{j}\right) P\left(x \mid w_{j}\right)$ is the evidence factor, and $P\left(w_{i} \mid x\right)$ is the posterior probability. The complete naive Bayesian classification model is the simplest Bayesian classification model. Its class subgraph and attribute subgraph are empty sets, and each class variable and attribute variable are connected by a directed edge. This method is based on a simple assumption that class variables are independent of each other. The multidimensional Bayesian model is defined as follows:

$$
\begin{aligned}
& \rho\left(c_{1}, c_{2}, \ldots, c_{m} \mid u_{1}, u_{2}, \ldots, u_{n}\right) \\
& \quad=\alpha \sum_{i=1}^{m} \sum_{j=1}^{n} \rho\left(c_{i}\right) \rho\left(u_{j} \mid c_{1}, c_{2}, \ldots, c_{m}\right) .
\end{aligned}
$$

$\alpha$ is the regularization parameter, $U=\left(u_{1}, u_{2}, \ldots, u_{n}\right)$ is unclassified data, and $c=\left(c_{1}, c_{2}, \ldots, c_{m}\right)$ is the arbitrary value of the class variable. We can achieve a relatively accurate result of classifications which can meet the practical application by using multidimensional Bayesian classifier.

3.4. Method Summary. Figure 2 is a sonar image processing flow chart. The sonar target automatic detection based on three-dimensional imaging includes a series of processes, such as sonar imaged preprocessing, noise exclusion, separation of water and stratum, image segmentation, and target discrimination. Using the sonar image preprocessing and SNR, we can effectively achieve the goal of denoising and reinforce. The image difference is so obvious owing to the differences between water and stratum that the OTSU algorithm is used to separate the water and stratum. Since water images generally contain less noise interference, we can choose OTSU algorithm and iterative threshold method for image segmentation. However, the noise of the stratum images is serious. To obtain a finer effect of segmentation, the iterative threshold method is a better choice. Then, we extract the characteristic values of the target, such as lengthwidth ratio, pixel value, and signal-to-noise ratio. Finally, the multidimensional Bayesian classification model is used to classify the target and realize the automatic detection of the whole sonar image. The flow chart is as in Figure 2.

\section{Experiment}

In order to verify the effectiveness and superiority of the proposed algorithm, this paper carries out simulation experiments on the three-dimensional imaging sonar data. The experimental data is all derived from Hangzhou Institute of Applied Acoustics. Firstly, check the noise suppression effect of sonar signal after bleaching process. Then the OTSU algorithm is used to separate the water and stratum so that the interface is found out. The image segmentation algorithm simulation experiment is carried out in the light of the characteristics of the sonar image of each part. The results of the two image segmentation methods are compared. The two methods are the iterative threshold method and the two-dimensional maximum entropy algorithm. Finally, the results of the classification are summarized by using the multidimensional Bayesian classification model.

4.1. Simulation Experiment of Sonar Signal Bleaching Process. Figure 3 is a comparison of the sonar signals before and after bleaching treatment. After the bleaching treatment, the background of the stratum's image is more balanced. Noise interference to a certain extent weakened which is conducive to the subsequent processing of the image. 

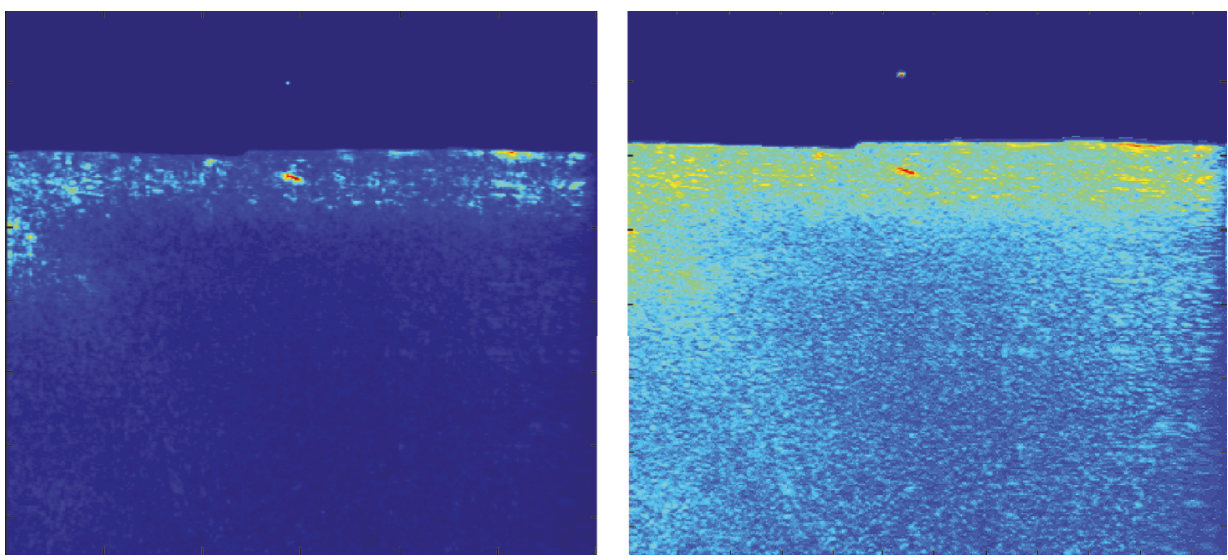

FIGURE 3: The comparison of the sonar signals before and after bleaching.
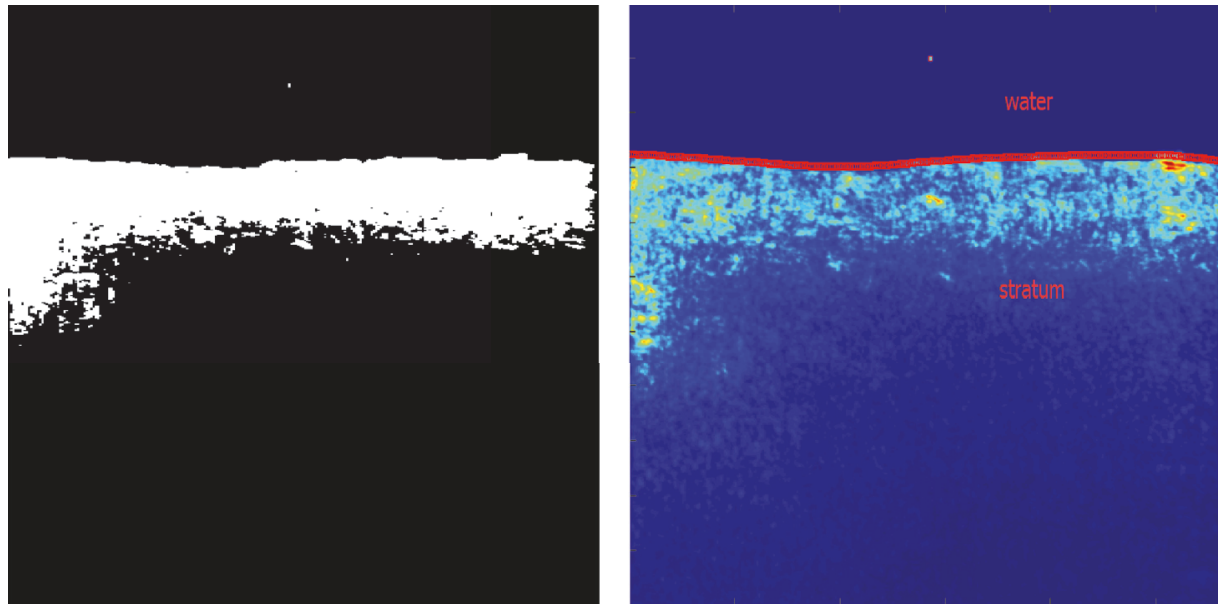

FIGURE 4: The results of water and stratum separation using OTSU.

4.2. Simulation Experiment of Water and Stratum Separation. Figure 4 is a graph of water and stratum separation; the left image is the OTSU binarized image. After binarization, it is clear to see the interface of water and stratum in the left image. We can effectively separate the water and stratum through mathematical morphology, such as corrosion expansion and opening and closing operations. The right image is the rendering of the segmentation. The red line in the figure is the interface, the upper part of the interface is the water, and the lower part is the stratum.

\subsection{Comparison of Iteration Threshold and 2D Maximum} Entropy Image Segmentation. Figures 5(a) and 5(b) are the images of the water and the stratum after image segmentation using the iterative threshold method. Figures 5(c) and 5(d) are the images of the water after image segmentation using the two-dimensional maximum entropy method. It is easy to find out that both the two methods can detect the spherical target in the water by the comparison of Figures 5(a) and 5(c), but the two-dimensional maximum entropy is easily disturbed by the interface. It can be seen from the comparison of Figures 5(b) and 5(d) that, in the segmentation of the stratum image, Figure 5(b) can better avoid the interference of the noise which is in favor of the detection of the target, but the noise interference is still more serious in the image using the twodimensional maximum entropy threshold. In summary, the iterative threshold method has a better effect.

4.4. Multidimensional Bayesian Classification Model and KNearest Neighbor Target Classification Contrast Test. For the object classification of the sonar image, the most commonly used methods are the K-nearest neighbor method and the multidimensional Bayesian classification model method. Although K-nearest neighbor method is simple and intelligible, its computational complexity is too high to apply to multiclassification tasks. In reality, submarine targets are different not only in shape but also in distribution. The multidimensional Bayesian classification model can accommodate smallscale data samples. At the same time, the multidimensional Bayesian classification model can adapt to multiclassification tasks and incremental training. Therefore, it is reasonable to use the multidimensional Bayesian classification model to classify the targets.

Figure 6 shows the experimental results using the Knearest neighbor method and the multidimensional Bayesian classification model within 40 images. Figure 7 is the 


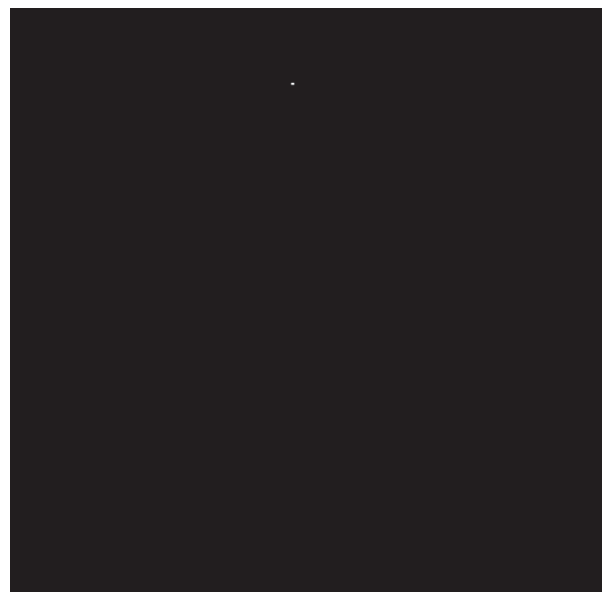

(a)

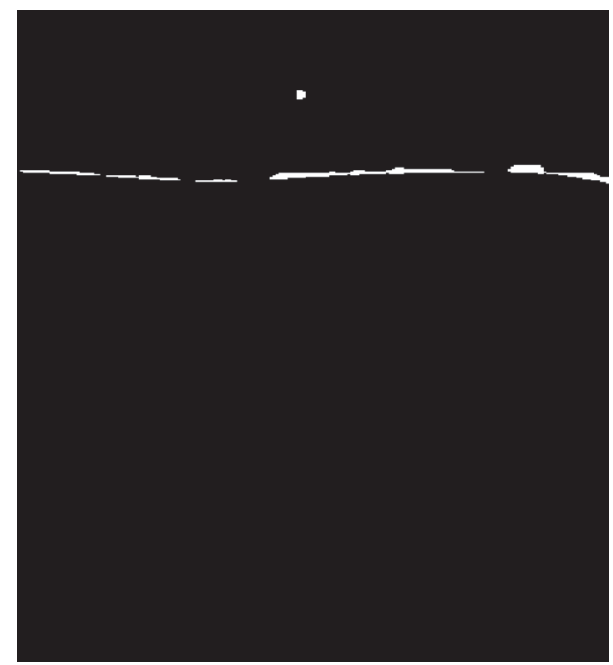

(c)

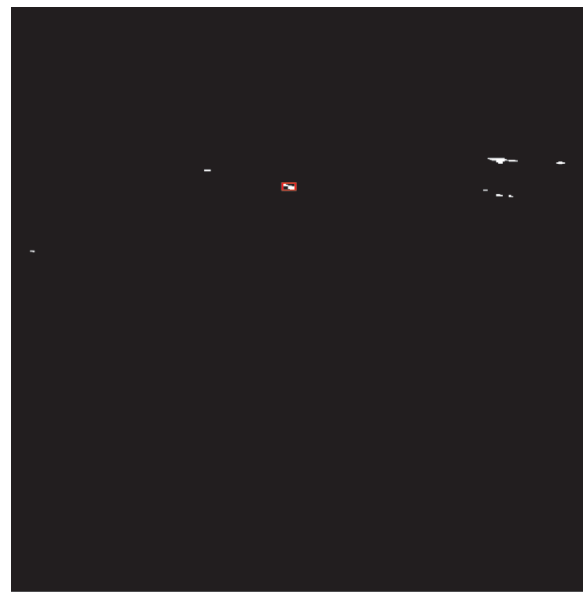

(b)

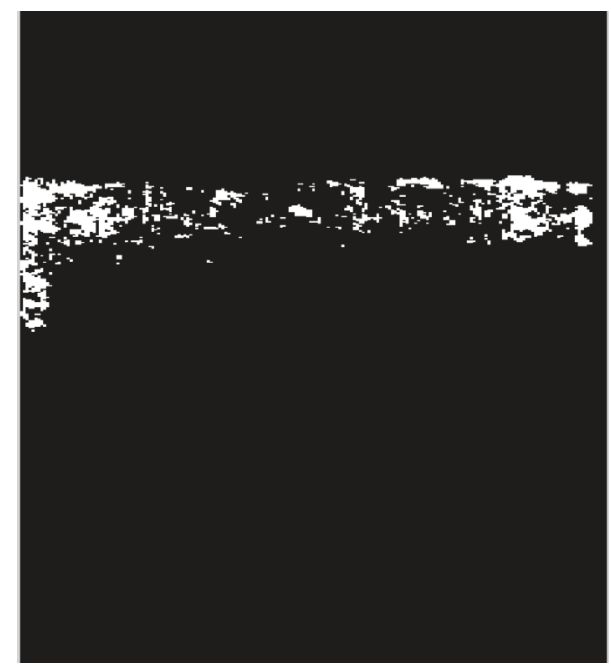

(d)

Figure 5: The comparison between the method we used and the two-dimensional maximum entropy.

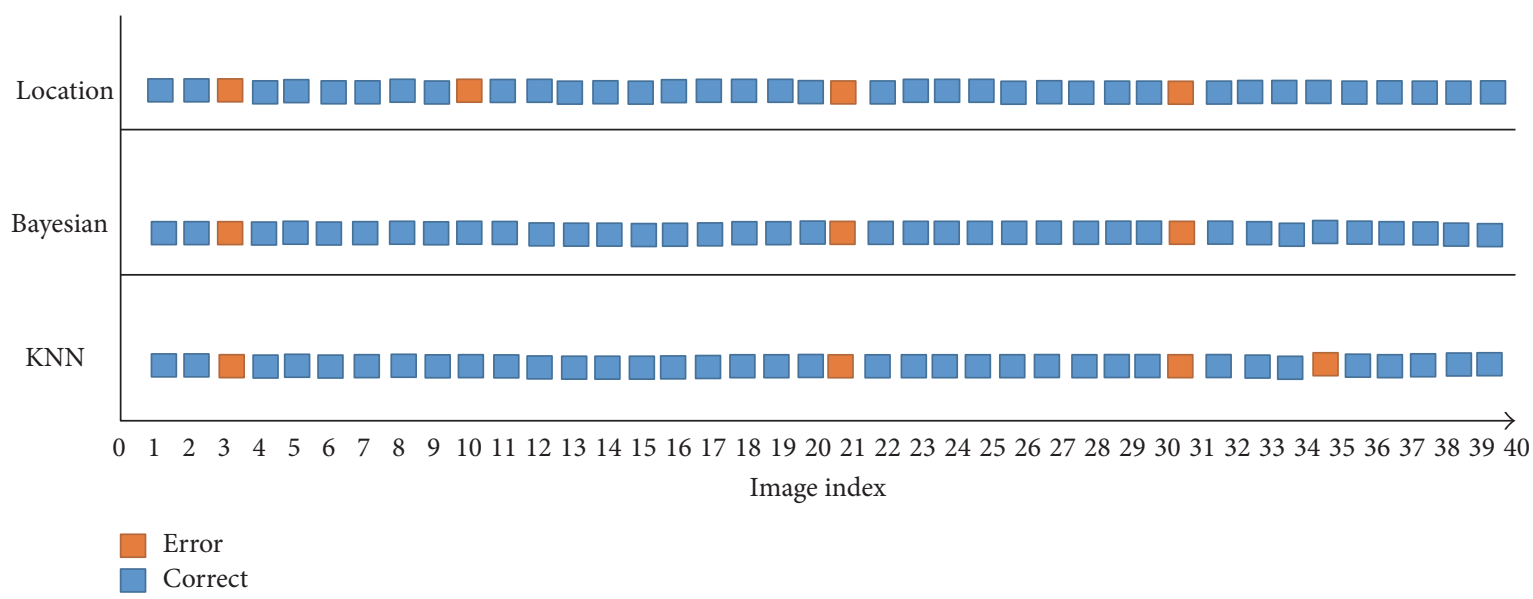

FIGURE 6: The classification result of the multidimensional Bayesian model and K-nearest neighbor method on a sonar dataset. 


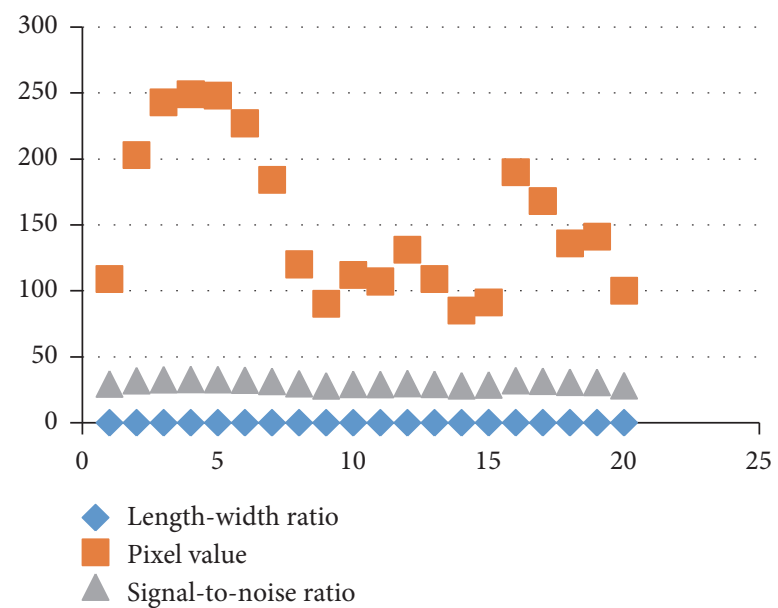

FIGURE 7: The characteristic distribution of columnar targets.

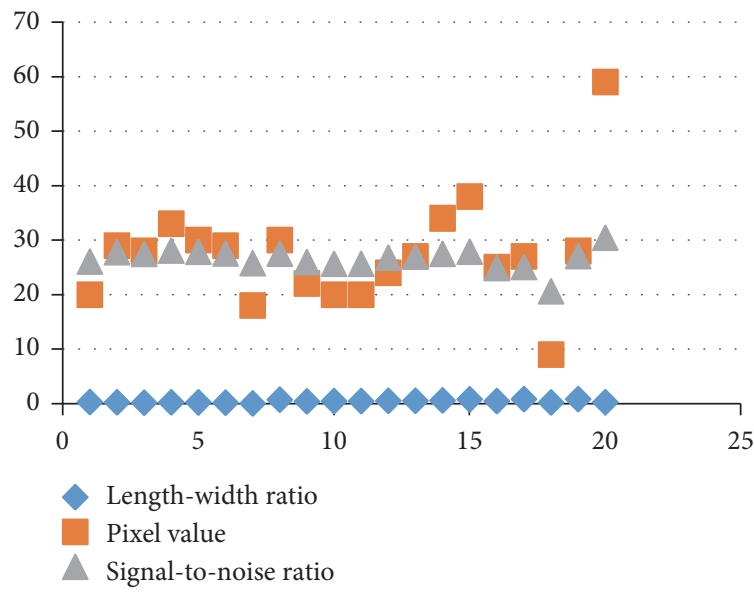

FIGURE 8: The characteristic distribution of spherical objects.

characteristic distribution of the columnar targets. Figure 8 is the characteristic distribution of the spherical targets. Classification of the columnar and spherical targets is based on the extracted length-width ratio, pixel value, and signalto-noise ratio. According to the experimental results of the two classification methods, there are only three misjudgments existing within the 40 sonar images by using the multidimensional Bayesian classification model. Otherwise, there are four misjudgments existing within the 40 sonar images by using the K-nearest neighbor method. It can be seen from the experimental results that the multidimensional Bayesian classification model and the K-nearest neighbor method can basically satisfy the requirements of the sonar image target classification because of the large differences in characteristics between the columnar and spherical target. However, given the small number of submarine target samples, the multidimensional Bayesian classifier can better meet the needs of target classification.

\section{Conclusions}

In this paper, we achieve the automatic detection technology of target in the sonar image based on three-dimensional imaging. This technology includes a complete set of processes such as sonar image preprocessing, water and stratum separation, image threshold segmentation, and target classification recognition. At the same time, a better sonar image processing result is achieved, which satisfies the actual processing requirements of the sonar image. Besides, this technology not only improves the efficiency of the sonar image target detection and reduces the unnecessary labor, but also improves accuracy of the sonar image target detection to a certain extent and creates a low false alarm rate and high detection rate of the sonar target detection technology. This technology has been applied in Hangzhou Institute of Applied Physics.

\section{Conflicts of Interest}

The authors declare that there are no conflicts of interest regarding the publication of this paper.

\section{Acknowledgments}

This work was supported by National Natural Science Foundation of China (Grant nos. 61671193 and 61102028), International Science \& Technology Cooperation Program of China (Grant no. 2014DFG12570), and project funded by China Postdoctoral Science Foundation (Grant no. 2015M571878).

\section{References}

[1] J. Luo, H. Liu, C. Huang, J. Gu, S. Xie, and H. Li, "Denoising and tracking of sonar video imagery for underwater security monitoring systems," in Proceedings of the IEEE International Conference on Robotics and Biomimetics (ROBIO '13), pp. 22032208, IEEE, Shenzhen, China, December 2013.

[2] I. Mandhouj, H. Amiri, F. Maussang, and B. Solaiman, "Sonar image processing for underwater object detection based on high resolution system," in Proceedings of the Workshop on Signal and Document Processing (SIDOP '12), vol. 845, Hammamet, Tunisia, March 2012.

[3] F. L. Casselman, D. F. Freeman, D. A. Kerrigan, S. E. Lane, N. H. Millstrom, and W. G. Nichols Jr., "A neural network-based passive sonar detection and classification design with a low false alarm rate," in Proceedings of the IEEE Conference on Neural Networks for Ocean Engineering, pp. 49-55, IEEE, Washington, DC, USA, August 1991.

[4] D. M. Weber and C. C. Kruger, "Detection of tonals in Lofargrams using connectionist methods," in Proceedings of the 1993 IEEE International Conference on Neural Networks, pp. 16621666, IEEE, San Francisco, Claif, USA, April 1993.

[5] B. P. Howell and S. Wood, "Passive sonar recognition and analysis using hybrid neural networks," in Proceedings of the IEEE Celebrating the Past-Teaming Toward the Future, pp. 1917-1924, San Diego, Claif, USA, September 2003.

[6] O. Postolache, P. Girao, and M. Pereira, "Underwater acoustic source localization based on passive sonar and intelligent processing," in Proceedings of the IEEE Instrumentation \& Measurement Technology Conference (IMTC '07), pp. 1-4, IEEE, Warsaw, Poland, May 2007. 
[7] D. C. Bertilone and D. S. Killeen, "Statistics of biological noise and performance of generalized energy detectors for passive detection," IEEE Journal of Oceanic Engineering, vol. 26, no. 2, pp. 285-294, 2001.

[8] J. Chen, Z. Gong, H. Li, and S. Xie, "A detection method based on sonar image for underwater pipeline tracker," in Proceedings of the 2nd International Conference on Mechanic Automation and Control Engineering (MACE '11), pp. 3766-3769, Hohhot, China, July 2011.

[9] H. Cho, J. Gu, H. Joe, A. Asada, and S.-C. Yu, "Acoustic beam profile-based rapid underwater object detection for an imaging sonar," Journal of Marine Science and Technology, vol. 20, no. 1, pp. 180-197, 2015.

[10] L.-Y. Weng, M. Li, Z. B. Gong, and S. G. Ma, "Underwater object detection and localization based on multi-beam sonar image processing," in Proceedings of the IEEE International Conference on Robotics and Biomimetics (ROBIO '12), pp. 514-519, IEEE, Guangzhou, China, December 2012.

[11] M. López, J. Ramírez, J. M. Górriz et al., "Automatic system for Alzheimer's disease diagnosis using eigenbrains and bayesian classification rules," in Bio-Inspired Systems: Computational and Ambient Intelligence, vol. 5517 of Lecture Notes in Computer Science, pp. 949-956, Springer Berlin Heidelberg, Berlin, Heidelberg, 2009.

[12] A.-A. Saucan, C. Sintes, T. Chonavel, and J.-M. Le Caillec, "Model-based adaptive 3D sonar reconstruction in reverberating environments," IEEE Transactions on Image Processing, vol. 24, no. 10, pp. 2928-2940, 2015.

[13] I. Mandhouj, F. Maussang, B. Solaiman, and H. Amiri, "Sonar image preprocessing method based on homomorphic filtering," in Proceedings of the OCEANS 2012 MTS/IEEE Hampton Roads Conference: Harnessing the Power of the Ocean, Hampton Roads, Va, USA, October 2012.

[14] X.-F. Ye, Z.-H. Zhang, P. X. Liu, and H.-L. Guan, "Sonar image segmentation based on GMRF and level-set models," Ocean Engineering, vol. 37, no. 10, pp. 891-901, 2010.

[15] Y. Han, "A detection method for underwater pipeline tracker based on sonar image," Computer Measurement \& Control, vol. 23, no. 2, pp. 539-541, 2015.

[16] H. B. Shi, Z. H. Wang, H. K. Huang et al., "A restricted doublelevel bayesian classification model," Journal of Software, vol. 15, no. 2, 2004. 


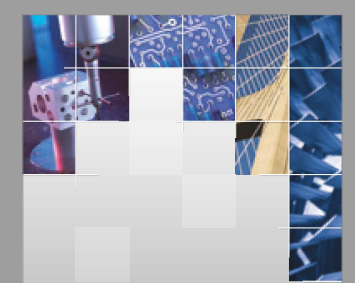

\section{Enfincering}
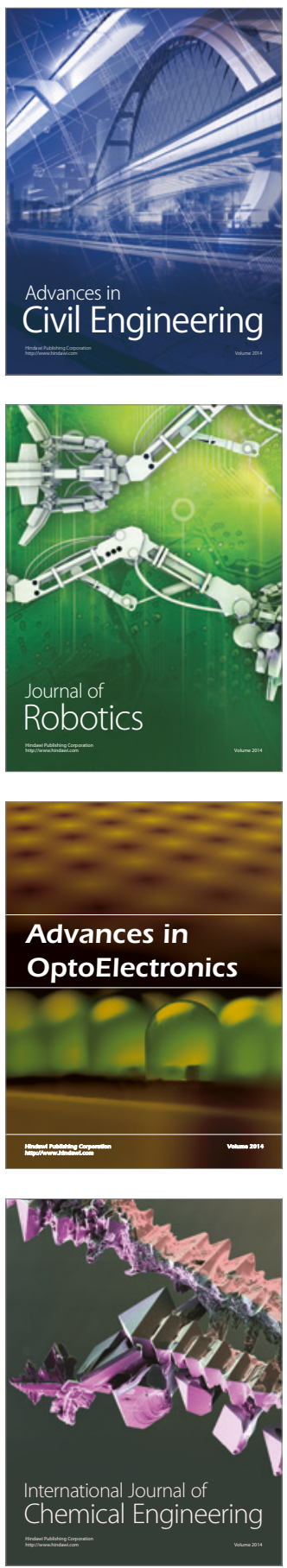

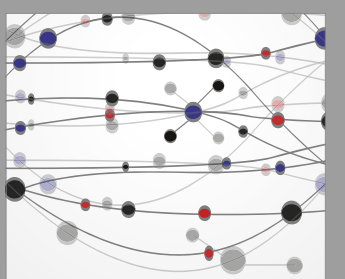

The Scientific World Journal

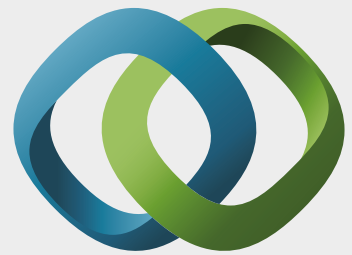

\section{Hindawi}

Submit your manuscripts at

https://www.hindawi.com
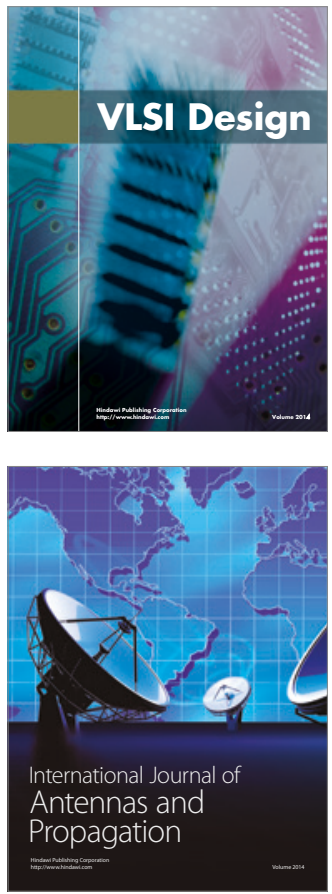

\section{Rotating}

Machinery
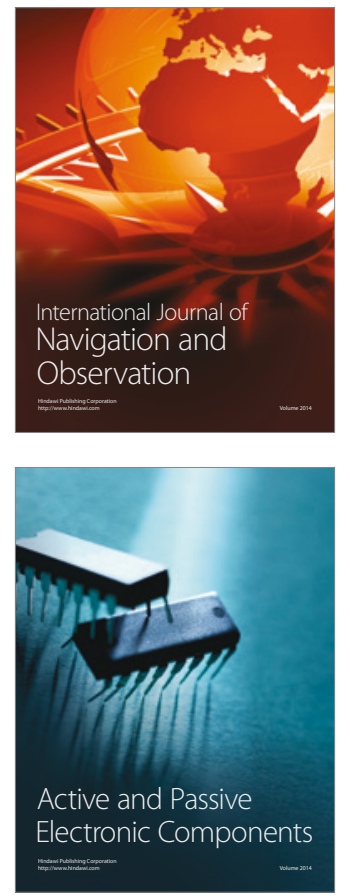
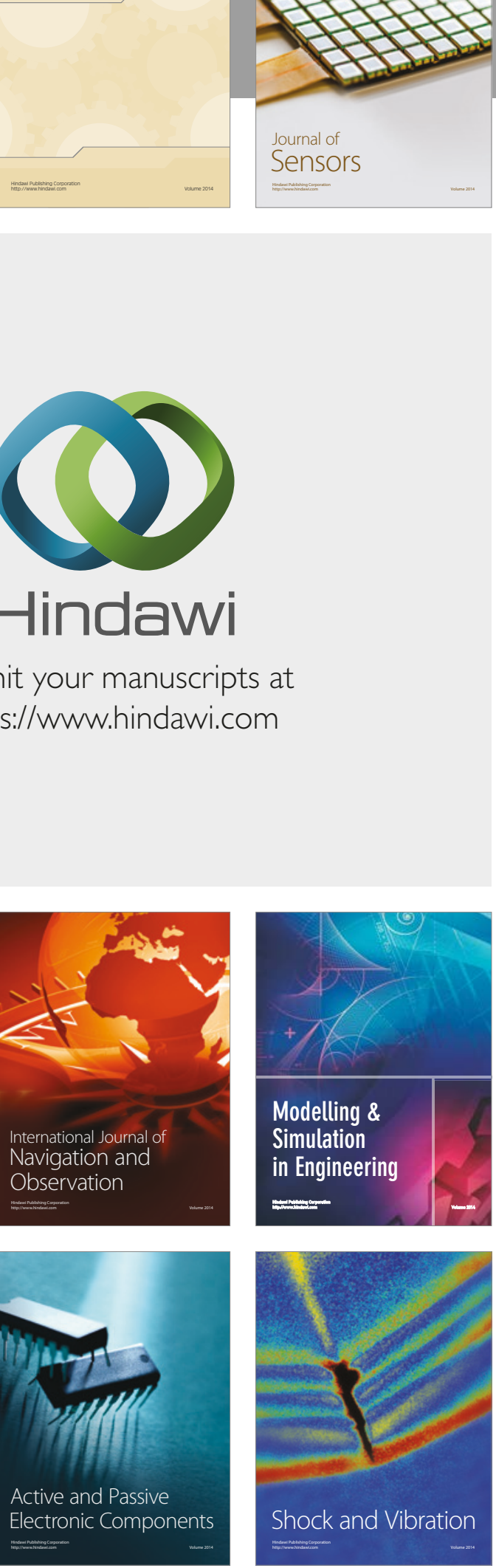
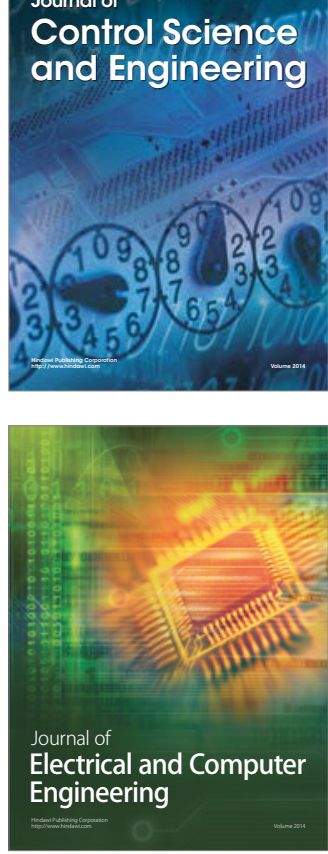

Distributed

Journal of

Control Science

and Engineering
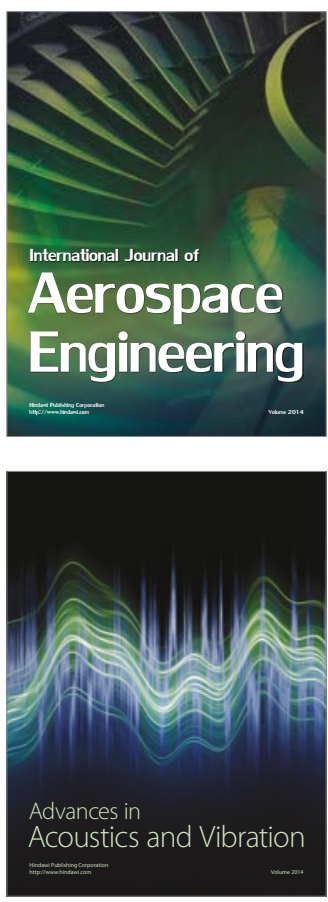

Sensor Networks 